\title{
Logarithmically Improved Regularity Criterion for the 3D Micropolar Fluid Equations
}

\author{
Hui Zhang \\ Department of Mathematics, Anqing Teachers College, Anqing 246011, China \\ Correspondence should be addressed to Hui Zhang; zhangaqtc@126.com
}

Received 9 November 2013; Accepted 31 December 2013; Published 13 February 2014

Academic Editor: Leo G. Rebholz

Copyright (C) 2014 Hui Zhang. This is an open access article distributed under the Creative Commons Attribution License, which permits unrestricted use, distribution, and reproduction in any medium, provided the original work is properly cited.

We study the regularity of weak solutions to the incompressible micropolar fluid equations. We obtain an improved regularity criterion in terms of vorticity of velocity in Besov space. It is proved that if the vorticity field satisfies $\int_{0}^{T}\left(\|\nabla \times u\|_{\dot{B}_{\infty, \infty}^{0}} /\right.$ $\left.\sqrt{1+\log \left(1+\|\nabla \times u\|_{\dot{B}_{\infty, \infty}^{0}}\right)}\right) d t<\infty$ then the strong solution can be smoothly extended after time $T$.

\section{Introduction}

This paper focuses on the incompressible micropolar fluid equations in $\mathbb{R}^{3}$

$$
\begin{gathered}
\partial_{t} u+(u \cdot \nabla) u-\Delta u+\nabla p-\nabla \times w=0, \\
\partial_{t} w-\Delta w-\nabla(\nabla \cdot w)+2 w+u \cdot \nabla w-\nabla \times u=0, \\
\nabla \cdot u=0, \\
u(x, 0)=u_{0}(x), \quad w(x, 0)=w_{0}(x),
\end{gathered}
$$

where $u(x, t)$ is the velocity field, $w(x, t)$ is the microrotational velocity field, and $p=p(x, t)$ is the scalar pressure field, while $\left(u_{0}, w_{0}\right)$ are the given initial data with $\nabla \cdot u_{0}=0$ in the sense of distribution.

Micropolar fluid system was firstly developed by Eringen $[1,2]$. It is a type of fluids which exhibits microrotational effects and microrotational inertia and can be viewed as a non-Newtonian fluid. It can describe many phenomena that appear in a large number of complex fluids such as the suspensions, animal blood, and liquid crystals which cannot be characterized appropriately by the Navier-Stokes system and that is important to the scientists working with the hydrodynamic-fluid problems and phenomena.

The existences of weak and strong solutions for micropolar fluid equations were treated by Galdi and Rionero [3] and Yamaguchi [4], respectively. The uniqueness of strong solutions to the micropolar flows and the magnetomicropolar flows either local for large data or global for small data is considered in $[5,6]$ and references therein.

The purpose of this paper is to study the regularity of weak solutions to the micropolar fluid system (1). By means of the Littlewood-Paley decomposition methods and function decomposition technique, Dong and Zhang [7, 8] recently prove the regularity of weak solutions under the velocity condition and the pressure condition in Besov spaces.

Yuan proved [9] some classical regularity criteria of weak solutions to the Navier-Stokes equation which also holds for the micropolar fluid equations. Particularly, the well-known Beale-Kato-Majda's criterion is also established [10].

If $(u, w)$ satisfies the condition

$$
\int_{0}^{T}\|\nabla \times u\|_{\dot{B}_{\infty, \infty}^{0}} d t<\infty
$$

then the solution $(u, w)$ can be extended smoothly beyond $t=T$.

Motivated by the ideas of [11-14], this paper is to establish logarithmically improved regularity criterion in terms of the vorticity. 
Theorem 1. Let $(u, w)$ be a smooth solution to (1) with initial data $\left(u_{0}, w_{0}\right) \in H^{3}\left(\mathbb{R}^{3}\right)$. Suppose that the corresponding vorticity field satisfies

$$
\int_{0}^{T} \frac{\|\nabla \times u\|_{\dot{B}_{\infty, \infty}^{0}}}{\sqrt{1+\log \left(1+\|\nabla \times u\|_{\dot{B}_{\infty, \infty}^{0}}\right)}} d t<\infty ;
$$

then the solution can be smoothly extended after time $T$.

We have the following corollary immediately.

Corollary 2. If the strong solution blows up at $T^{*}$, then

$$
\int_{0}^{T^{*}} \frac{\|\nabla \times u\|_{\dot{B}_{\infty, \infty}^{0}}}{\sqrt{1+\log \left(1+\|\nabla \times u\|_{\dot{B}_{\infty, \infty}^{0}}\right)}} d t=\infty .
$$

Remark 3. Theorem 1 can be regarded as an extension of [11] to $3 \mathrm{D}$ Navier-Stokes equations.

Now, we recall the definition of weak solutions for micropolar fluid equations.

Definition 4. Let $\left(u_{0}, w_{0}\right) \in L^{2}\left(\mathbb{R}^{3}\right)$ and $\nabla \cdot u_{0}=0$ in the sense of distribution. A pair vector field $(u, w)$ is termed as a weak solution of $(1)$ on $(0, T)$, if it satisfies the following conditions:

(1) $(u, w) \in L^{\infty}\left(0, T ; L^{2}\left(\mathbb{R}^{3}\right)\right) \cap L^{2}\left(0, T ; H^{1}\left(\mathbb{R}^{3}\right)\right)$;

(2) $(u, w)$ verifies (1) in the sense of distribution;

(3) $\operatorname{div} u=0$ in the sense of distribution.

By a strong solution we mean a weak solution $(u, w)$ of micropolar fluid equations (1) with the initial velocity $\left(u_{0}, w_{0}\right) \in H^{1}\left(\mathbb{R}^{3}\right)$ which satisfies

$$
(u, w) \in L^{\infty}\left(0, T ; H^{1}\left(\mathbb{R}^{3}\right)\right) \cap L^{2}\left(0, T ; H^{2}\left(\mathbb{R}^{3}\right)\right) .
$$

It is well known that strong solutions are regular and unique.

Remark 5. Throughout the paper, $C$ stands for a constant and changes from line to line; $\|\cdot\|_{p}$ denotes the norm of the Lebesgue space $L^{p}\left(\mathbb{R}^{3}\right)$ and $\|\cdot\|_{H^{p}}$ denotes the norm of the Lebesgue space $H^{p}\left(\mathbb{R}^{3}\right)$.

\section{Proof of Theorem 1}

Before going to the proof, we recall the following two inequalities established in $[15,16]$.

Lemma 6. Let $1<r<\infty$. Then we have

$$
\begin{aligned}
\| f & \cdot \nabla g \|_{r} \\
& \leq C\left(\|f\|_{r}\left\|(-\Delta)^{1 / 2} g\right\|_{\mathrm{BMO}}+\left\|(-\Delta)^{1 / 2} f\right\|_{\mathrm{BMO}}\|g\|_{r}\right)
\end{aligned}
$$

for all $f, g \in W^{1, r}\left(\mathbb{R}^{3}\right)$ with $\nabla f, \nabla g \in \mathrm{BMO}$ with $C=C(n, r)$.
Lemma 7. Let $p, q, \rho, \sigma, v \in[1, \infty]$ with $v \leq \min (\rho, \sigma), 1 / q=$ $1 / p-s / n, 1 \leq r \leq q$, and $s_{1} / n<1 / r-1 / q<s_{2} / n$. Then, for $f \in \dot{B}_{r, \sigma}^{s_{1}} \cap \dot{B}_{r, \sigma}^{s_{2}}$, we have

$$
\begin{aligned}
& \|f\|_{\dot{B}_{q, v}^{0}} \\
& \quad \leq C\left(1+\|f\|_{\dot{B}_{p, \rho}^{s}}\left(\log ^{+}\left(\|f\|_{\dot{B}_{r, \sigma}^{s_{1}}}+\|f\|_{\dot{B}_{r, \sigma}^{s_{2}}}\right)\right)^{(1 / v)-(1 / \rho)}\right) .
\end{aligned}
$$

By choosing $p=q=\rho=\infty, \nu=r=\sigma=s_{2}=2$, and $s_{1}=s=0$, we have

$$
\|f\|_{\text {BMO }} \leq C\left(1+\|f\|_{\dot{B}_{\infty, \infty}^{0}} \log ^{1 / 2}\left(1+\|f\|_{H^{2}}\right)\right),
$$

where we used the following relations:

$$
\begin{gathered}
\dot{B}_{\infty, 2}\left(\mathbb{R}^{3}\right) \subset \dot{F}_{\infty, 2}\left(\mathbb{R}^{3}\right)=\text { BMO, } \\
\dot{B}_{2,2}^{s}\left(\mathbb{R}^{3}\right)=\dot{H}^{s}\left(\mathbb{R}^{3}\right) .
\end{gathered}
$$

Proof of Theorem 1. Multiplying the first equation of (1) by $|u|^{2} u$, after integration by parts, we have

$$
\begin{aligned}
& \frac{1}{4} \frac{d}{d t}\|u\|_{4}^{4}+\frac{1}{2}\left\|\nabla|u|^{2}\right\|_{2}^{2}+\int_{\mathbb{R}^{3}}|u|^{2}|\nabla u|^{2} d x \\
& \quad=\int_{\mathbb{R}^{3}}(\nabla \times w)|u|^{2} u d x-\int_{\mathbb{R}^{3}} \nabla p \cdot u|u|^{2} d x .
\end{aligned}
$$

Similarly, multiplying the second equation of (1) by $|w|^{2} w$, we obtain

$$
\begin{aligned}
& \frac{1}{4} \frac{d}{d t}\|w\|_{4}^{4}+\frac{1}{2}\left\|\nabla|w|^{2}\right\|_{2}^{2} \\
& \quad+\int_{\mathbb{R}^{3}}|w|^{2}|\nabla w|^{2} d x+\||w| \operatorname{div} w\|_{2}^{2}+2\|w\|_{4}^{4} \\
& \leq \int_{\mathbb{R}^{3}} \nabla \times u \cdot\left(|w|^{2} w\right) d x+\int_{\mathbb{R}^{3}} \operatorname{div} w \cdot w \nabla|w|^{2} d x .
\end{aligned}
$$

Adding (10) to (11), one has that

$$
\begin{aligned}
& \frac{1}{4}\left(\frac{d}{d t}\|w\|_{4}^{4}+\frac{d}{d t}\|u\|_{4}^{4}\right)+\frac{1}{2}\left(\left\|\nabla|w|^{2}\right\|_{2}^{2}+\left\|\nabla|u|^{2}\right\|_{2}^{2}\right) \\
& \quad+\left(\||w||\nabla w|\|_{2}^{2}+\||u||\nabla u|\|_{2}^{2}\right) \\
& \quad+\||w| \operatorname{div} w\|_{2}^{2}+2\|w\|_{4}^{4} \\
& \leq \int_{\mathbb{R}^{3}}(\nabla \times w)|u|^{2} u d x-\int_{\mathbb{R}^{3}} \nabla p \cdot u|u|^{2} d x \\
& \quad+\int_{\mathbb{R}^{3}} \nabla \times u \cdot\left(|w|^{2} w\right) d x+2 \int_{\mathbb{R}^{3}} \operatorname{div} w|w| w \nabla|w| d x \\
& =I_{1}+I_{2}+I_{3}+I_{4} .
\end{aligned}
$$

We estimate above terms one by one, using the following relation:

$$
\nabla \cdot(A \times B)=B \cdot \nabla \times A-A \cdot \nabla \times B .
$$


We have

$$
\begin{aligned}
I_{1} & =\int_{\mathbb{R}^{3}} w \nabla \times\left(|u|^{2} u\right) d x \\
& \leq \int_{\mathbb{R}^{3}}|w||u|^{2}(|\nabla u|+2|\nabla| u||) d x \\
& \leq 3 \int_{\mathbb{R}^{3}}|w||u|^{2}|\nabla u| d x,
\end{aligned}
$$

where we have used the fact $|\nabla| u|| \leq|\nabla u|$.

Applying Holder inequality and Cauchy inequality, we get

$$
I_{1} \leq C\left(\|w\|_{4}^{4}+\|u\|_{4}^{4}\right)+\frac{3}{4}\||u| \mid \nabla u\|_{2}^{2} .
$$

Similarly, for $I_{3}$, we have

$$
I_{3} \leq C\left(\|w\|_{4}^{4}+\|u\|_{4}^{4}\right)+\frac{3}{4}\||w||\nabla w|\|_{2}^{2} .
$$

In the same way, for $I_{4}$ one can deduce

$$
\begin{aligned}
I_{4} & \leq\left.\int_{\mathbb{R}^{3}}|\operatorname{div} w| w|||\nabla| w\right|^{2} \mid d x \\
& \leq\|\operatorname{div} w|w|\|_{2}^{2}+\frac{1}{4}\left\|\nabla|w|^{2}\right\|_{2}^{2} .
\end{aligned}
$$

In order to estimate $I_{2}$, we first establish an estimate between the pressure and the velocity. Taking the operator div on both sides of the first equation of (1),

$$
p=(-\Delta)^{-1} \sum_{i, j=1}^{3} \partial_{i} \partial_{j}\left(u_{i} u_{j}\right)
$$

Applying $L^{p}(1<p<\infty)$ boundedness of the singular operators yields

$$
\|\nabla p\|_{p} \leq C\|(u \cdot \nabla) u\|_{p}
$$

Inequality (19), together with Lemma 6 , shows that

$$
\begin{aligned}
I_{2} & \leq\|\nabla p\|_{4}\|u\|_{4}^{3} \leq C\|u \cdot \nabla u\|_{4}\|u\|_{4}^{3} \\
& \leq C\|u\|_{4}^{4}\|\nabla u\|_{\text {BMO }} .
\end{aligned}
$$

Combining (12), (15), (16), (17), and (20) yields

$$
\frac{d}{d t}\left(\|w\|_{4}^{4}+\|u\|_{4}^{4}\right) \leq C\|\nabla u\|_{\mathrm{BMO}}\left(\|w\|_{4}^{4}+\|u\|_{4}^{4}\right) .
$$

For the right hand side of (21), we have

$$
\begin{array}{r}
\|\nabla u\|_{\text {BMO }} \\
\leq C\left(1+\|\nabla u\|_{\dot{B}_{\infty, \infty}^{0}} \sqrt{\log \left(1+\|\nabla u\|_{H^{2}}\right)}\right) \\
\leq C\left(1+\frac{\|\nabla u\|_{\dot{B}_{\infty, \infty}^{0}}}{\sqrt{1+\log \left(1+\|\nabla \times u\|_{\dot{B}_{\infty, \infty}^{0}}\right)}}\right. \\
\left.\quad \times\left(1+\log \left(1+\left\|\nabla^{3} u\right\|_{2}\right)\right)\right)
\end{array}
$$

$$
\leq C\left(1+\frac{\|\nabla \times u\|_{\dot{B}_{\infty, \infty}^{0}}}{\sqrt{1+\log \left(1+\|\nabla \times u\|_{\dot{B}_{\infty, \infty}^{0}}\right)}}\right.
$$

$$
\left.\times\left(1+\log \left(1+\left\|\nabla^{3} u\right\|_{2}\right)\right)\right),
$$

where we used the inequality $\|\nabla u\|_{\dot{B}_{\infty, \infty}^{0}} \leq C\|\nabla \times u\|_{\dot{B}_{\infty, \infty}^{0}}$.

Due to (3), one can show that, for any small constant $\epsilon>$ 0 , there exists $T_{*}<T$ such that

$$
\int_{T_{*}}^{T} \frac{\|\nabla \times u\|_{\dot{B}_{\infty, \infty}^{0}}}{\sqrt{1+\log \left(1+\|\nabla \times u\|_{\dot{B}_{\infty, \infty}^{0}}\right)}} d t<\epsilon .
$$

For any $T_{*}<t \leq T$, we set

$$
y(t)=\sup _{T_{*} \leq s \leq t}\left(\left\|\nabla^{3} u\right\|_{2}+\left\|\nabla^{3} w\right\|_{2}\right) .
$$

Applying Gronwall's inequality to $(21)$ in the interval $\left[T_{*}, t\right]$, one has

$$
\left(\|w\|_{4}^{4}+\|u\|_{4}^{4}\right) \leq C_{*}(1+y(t))^{C \epsilon},
$$

where $C_{*}$ is a positive constant depending on $T_{*}$ and $\left\|w\left(\cdot, T_{*}\right)\right\|_{4}^{4}+\left\|u\left(\cdot, T_{*}\right)\right\|_{4}^{4}$. 
Applying $\nabla$ to (1) and taking the $L^{2}$ inner product of the resulting equation with $(\nabla u, \nabla w)$ with help of integrating by parts, we have

$$
\begin{aligned}
\frac{1}{2} \frac{d}{d t}( & \left.\|\nabla u\|_{2}^{2}+\|\nabla w\|_{2}^{2}\right)+\left(\left\|\nabla^{2} u\right\|_{2}^{2}+\left\|\nabla^{2} w\right\|_{2}^{2}\right) \\
& +\|\nabla \nabla \cdot w\|_{2}^{2}+2\|\nabla w\|_{2}^{2} d x \\
= & -\int_{R^{3}} \nabla[(u \cdot \nabla) u] \nabla u d x-\int_{R^{3}} \nabla[(u \cdot \nabla) w] \nabla w d x \\
& +\int_{R^{3}} \nabla(\nabla \times w) \nabla u d x+\int_{R^{3}} \nabla(\nabla \times u) \nabla w d x \\
= & \sum_{i=1}^{4} K_{i} .
\end{aligned}
$$

To estimate $K_{1}$, we integrate by parts and apply Holder's inequality to obtain

$$
\begin{aligned}
K_{1} & =-\int_{\mathbb{R}^{3}} \nabla[(u \cdot \nabla) u] \nabla u d x \\
& =-\sum_{i, j, k=1}^{3} \int_{\mathbb{R}^{3}} \partial_{k}\left(u_{i} \partial_{i} u_{j}\right) \partial_{k} u_{j} d x \\
& =\sum_{i, j, k=1}^{3} \int_{R^{3}} u_{i} \partial_{i} u_{j} \partial_{k k} u_{j} d x \\
\leq & C\|u\|_{4}\|\nabla u\|_{4}\left\|\nabla^{2} u\right\|_{2} \\
\leq & C\|u\|_{4}^{8}\|\nabla u\|_{2}^{2}+\frac{1}{4}\left\|\nabla^{2} u\right\|_{2} \cdot \\
K_{2}= & -\int_{R^{3}} \nabla[(u \cdot \nabla) w] \nabla w d x \\
= & -\sum_{i, j, k=1}^{3} \int_{\mathbb{R}^{3}} \partial_{k}\left(u_{i} \partial_{i} w_{j}\right) \partial_{k} w_{j} d x \\
= & -\sum_{i, j, k=1}^{3} \int_{\mathbb{R}^{3}} \partial_{k} u_{i} \partial_{i} w_{j} \partial_{k} w_{j} d x \\
& -\sum_{i, j, k=1}^{3} \int_{\mathbb{R}^{3}} u_{i} \partial_{i} \partial_{k} w_{j} \partial_{k} w_{j} d x \\
= & \sum_{i, j, k=1}^{3} \int_{\mathbb{R}^{3}} u_{i} \partial_{k}\left(\partial_{i} w_{j} \partial_{k} w_{j}\right) d x \\
& -\sum_{i, j, k=1}^{3} \int_{\mathbb{R}^{3}} u_{i} \partial_{i} \partial_{k} w_{j} \partial_{k} w_{j} d x=K_{21}+K_{22} .
\end{aligned}
$$

By Holder's inequality and Young's inequality, we have

$$
\left|K_{21}\right| \leq C\|u\|_{4}^{8}\|\nabla w\|_{2}^{2}+\frac{1}{4}\left\|\nabla^{2} w\right\|_{2}^{2} .
$$

Due to the incompressible condition $\nabla \cdot u=0$, we obtain

$$
\left|K_{22}\right|=0 .
$$

By integrating by parts and applying Holder's inequality and Young's inequality, we have

$$
\begin{aligned}
& \left|K_{3}+K_{4}\right| \\
& \quad=\left|\int_{\mathbb{R}^{3}} \nabla(\nabla \times w) \nabla u+\nabla(\nabla \times u) \nabla w d x\right| \\
& \quad \leq \int_{\mathbb{R}^{3}}\left|\nabla^{2} u\right||\nabla w| d x \leq \frac{1}{4}\left\|\nabla^{2} u\right\|_{2}^{2}+\|\nabla w\|_{2}^{2} .
\end{aligned}
$$

From the above computation, we have

$$
\begin{aligned}
\frac{d}{d t}( & \left(\|\nabla u\|_{2}^{2}+\|\nabla w\|_{2}^{2}\right)+\left(\left\|\nabla^{2} u\right\|_{2}^{2}+\left\|\nabla^{2} w\right\|_{2}^{2}\right) \\
& +\|\nabla \nabla \cdot w\|_{2}^{2}+2\|\nabla w\|_{2}^{2} d x \\
\leq & C\|u\|_{4}^{8}\left(\|\nabla u\|_{2}^{2}+\|\nabla w\|_{2}^{2}\right) \\
\leq & C\|u\|_{4}^{16}+C\left(\|\nabla u\|_{2}^{2}+\|\nabla w\|_{2}^{2}\right)^{2} .
\end{aligned}
$$

Applying Gronwall's inequality and (25), we have

$$
\sup _{T_{*} \leq s \leq t}\left(\|\nabla u\|_{2}^{2}+\|\nabla w\|_{2}^{2}\right) \leq C+C_{*}(1+y(t))^{C \epsilon} .
$$

We should point out that the constant $C_{*}$ also changes from line to line.

Applying $\nabla^{3}$ to (1) and taking the $L^{2}$ inner product of the resulting equation with $\left(\nabla^{3} u, \nabla^{3} w\right)$ with help of integrating by parts, we have

$$
\begin{aligned}
\frac{1}{2} \frac{d}{d t} & \left(\left\|\nabla^{3} u\right\|_{2}^{2}+\left\|\nabla^{3} w\right\|_{2}^{2}\right)+\left(\left\|\nabla^{4} u\right\|_{2}^{2}+\left\|\nabla^{4} w\right\|_{2}^{2}\right) \\
& +\left\|\nabla^{3} \nabla \cdot w\right\|_{2}^{2}+2\left\|\nabla^{3} w\right\|_{2}^{2} \\
= & -\int_{R^{3}} \nabla^{3}[(u \cdot \nabla) u] \nabla^{3} u d x \\
& -\int_{R^{3}} \nabla^{3}[(u \cdot \nabla) w] \nabla^{3} w d x \\
& +\int_{R^{3}} \nabla^{3}(\nabla \times w) \nabla^{3} u d x+\int_{R^{3}} \nabla^{3}(\nabla \times u) \nabla^{3} w d x \\
= & \sum_{i=1}^{4} H_{i} .
\end{aligned}
$$

Now, we introduce the following commutator estimate according to Kato and Ponce [17]:

$$
\begin{aligned}
& \left\|\nabla^{\alpha}(f g)-f \nabla^{\alpha} g\right\|_{p} \\
& \quad \leq C\left(\left\|\nabla^{\alpha-1} g\right\|_{q_{1}}\|f\|_{p_{1}}+\left\|\nabla^{\alpha} f\right\|_{q_{2}}\|g\|_{p_{2}}\right)
\end{aligned}
$$


for $\alpha>1$ and $1 / p=1 / p_{1}+1 / q_{1}=1 / p_{2}+1 / q_{2}$. One finds

$$
\begin{aligned}
\left|H_{1}\right| & \leq C\left\|\nabla^{3} u\right\|_{3}^{2}\|\nabla u\|_{3} \leq C\|\nabla u\|_{2}^{13 / 12}\left\|\nabla^{3} u\right\|_{2}^{1 / 4}\left\|\nabla^{4} u\right\|_{2}^{5 / 3} \\
& \leq C\|\nabla u\|_{2}^{13 / 2}\left\|\nabla^{3} u\right\|_{2}^{3 / 2}+\frac{1}{8}\left\|\nabla^{4} u\right\|_{2}^{2} \\
& \leq C_{*}(1+y(t))^{(3 / 2)+(13 / 2) C \epsilon}+\frac{1}{8}\left\|\nabla^{4} u\right\|_{2}^{2}
\end{aligned}
$$

where we used the following inequalities

$$
\begin{gathered}
\|\nabla u\|_{3} \leq C\|\nabla u\|_{2}^{3 / 4}\left\|\nabla^{3} u\right\|_{2}^{1 / 4} \\
\left\|\nabla^{3} u\right\|_{3} \leq C\|\nabla u\|_{2}^{1 / 6}\left\|\nabla^{4} u\right\|_{2}^{5 / 6}
\end{gathered}
$$

Similarly, we can do estimate for $\mathrm{H}_{2}$ as follows

$$
\begin{aligned}
\left|H_{2}\right| \leq & \left(\|\nabla u\|_{3}\left\|\nabla^{3} w\right\|_{3}+\left\|\nabla^{3} u\right\|_{3}\|\nabla w\|_{3}\right)\left\|\nabla^{3} w\right\|_{3} \\
= & \|\nabla u\|_{3}\left\|\nabla^{3} w\right\|_{3}^{2}+\left\|\nabla^{3} u\right\|_{3}\|\nabla w\|_{3}\left\|\nabla^{3} w\right\|_{3} \\
= & H_{21}+H_{22} \\
H_{21}= & \|\nabla u\|_{3}\left\|\nabla^{3} w\right\|_{3}^{2} \\
\leq & C\|\nabla u\|_{2}^{3 / 4}\left\|\nabla^{3} u\right\|_{2}^{1 / 4}\|\nabla w\|_{2}^{1 / 3}\left\|\nabla^{4} w\right\|_{2}^{5 / 3} \\
\leq & C\|\nabla u\|_{2}^{9 / 2}\left\|\nabla^{3} u\right\|_{2}^{3 / 2}\|\nabla w\|_{2}^{2}+\frac{3}{8}\left\|\nabla^{3} w\right\|_{2}^{2} \\
\leq & C *(1+y(t))^{(3 / 2)+(13 / 2) C \epsilon}+\frac{3}{8}\left\|\nabla^{4} w\right\|_{2}^{2} \\
H_{22}= & \left\|\nabla^{3} u\right\|_{3}\|\nabla w\|_{3}\left\|\nabla^{3} w\right\|_{3} \\
\leq & C\|\nabla w\|_{3}\|\nabla w\|_{2}^{1 / 3}\left\|\nabla^{4} w\right\|_{2}^{5 / 3} \\
& +\|\nabla w\|_{3}\|\nabla u\|_{2}^{1 / 3}\left\|\nabla^{4} u\right\|_{2}^{5 / 3} \\
\leq & C\left\|\nabla^{3} w\right\|_{2}^{3 / 2}\left(\|\nabla w\|_{2}^{13 / 2}+\|\nabla w\|_{2}^{9 / 2}\|\nabla u\|_{2}^{2}\right) \\
& +\frac{1}{8}\left(\left\|\nabla^{4} w\right\|_{2}^{2}+\left\|\nabla^{4} u\right\|_{2}^{2}\right) \\
& =y(t))^{(3 / 2)+(13 / 2) C \epsilon}+\frac{1}{8}\left(\left\|\nabla^{4} w\right\|_{2}^{2}+\left\|\nabla^{4} u\right\|_{2}^{2}\right) .
\end{aligned}
$$

By integrating by parts and applying Holder's inequality and Young's inequality, we have

$$
\begin{aligned}
& \left|H_{3}+H_{4}\right| \\
& \quad=\left|\int_{R^{3}} \nabla^{3}(\nabla \times w) \nabla^{3} u+\nabla^{3}(\nabla \times u) \nabla^{3} w d x\right| \\
& \quad \leq \int_{R^{3}}\left|\nabla^{4} u\right|\left|\nabla^{3} w\right| d x \leq \frac{1}{4}\left\|\nabla^{2} u\right\|_{2}^{2}+\left\|\nabla^{3} w\right\|_{2}^{2} .
\end{aligned}
$$

Combining (33)-(38), it follows that

$$
\frac{d}{d t}\left(\left\|\nabla^{3} u\right\|_{2}^{2}+\left\|\nabla^{3} w\right\|_{2}^{2}\right) \leq C_{*}(1+y(t))^{(3 / 2)+(13 / 2) C \epsilon} .
$$

It should be clear that applying Gronwall's inequality to (39), we can obtain $y(t) \leq C$ provided that $\epsilon$ is small enough. This completes the proof of the theorem.

\section{Conflict of Interests}

The author declares that there is no conflict of interests regarding the publication of this paper.

\section{References}

[1] A. C. Eringen, “Theory of micropolar fluids," Journal of Mathematics and Mechanics, vol. 16, no. 1, pp. 1-18, 1966.

[2] A. C. Eringen, "Simple microfluids," International Journal of Engineering Science, vol. 2, pp. 205-217, 1964.

[3] G. P. Galdi and S. Rionero, "A note on the existence and uniqueness of solutions of the micropolar fluid equations," International Journal of Engineering Science, vol. 15, no. 2, pp. 105-108, 1977.

[4] N. Yamaguchi, "Existence of global strong solution to the micropolar fluid system in a bounded domain," Mathematical Methods in the Applied Sciences, vol. 28, no. 13, pp. 1507-1526, 2005.

[5] J. L. Boldrini, M. A. Rojas-Medar, and E. Fernández-Cara, "Semi-Galerkin approximation and strong solutions to the equations of the nonhomogeneous asymmetric fluids," Journal de Mathématiques Pures et Appliquées, vol. 82, no. 11, pp. 14991525, 2003.

[6] M. A. Rojas-Medar, "Magneto-micropolar fluid motion: existence and uniqueness of strong solution," Mathematische Nachrichten, vol. 188, pp. 301-319, 1997.

[7] B.-Q. Dong and W. Zhang, "On the regularity criterion for three-dimensional micropolar fluid flows in Besov spaces," Nonlinear Analysis: Theory, Methods \& Applications, vol. 73, no. 7, pp. 2334-2341, 2010.

[8] Y. Jia, W. Zhang, and B.-Q. Dong, "Remarks on the regularity criterion of the 3D micropolar fluid flows in terms of the pressure," Applied Mathematics Letters, vol. 24, no. 2, pp. 199203, 2011.

[9] B. Yuan, "On regularity criteria for weak solutions to the micropolar fluid equations in Lorentz space," Proceedings of the American Mathematical Society, vol. 138, no. 6, pp. 2025-2036, 2010.

[10] B. Yuan, "Regularity of weak solutions to magneto-micropolar fluid equations," Acta Mathematica Scientia B, vol. 30, no. 5, pp. 1469-1480, 2010.

[11] J. Fan, S. Jiang, G. Nakamura, and Y. Zhou, "Logarithmically improved regularity criteria for the Navier-Stokes and MHD equations," Journal of Mathematical Fluid Mechanics, vol. 13, no. 4, pp. 557-571, 2011.

[12] Y. Zhou and S. Gala, "Logarithmically improved regularity criteria for the Navier-Stokes equations in multiplier spaces," Journal of Mathematical Analysis and Applications, vol. 356, no. 2, pp. 498-501, 2009.

[13] Y. Zhou and J. Fan, "Logarithmically improved regularity criteria for the 3D viscous MHD equations," Forum Mathematicum, vol. 24, no. 4, pp. 691-708, 2012. 
[14] J. Fan, Y. Fukumoto, and Y. Zhou, "Logarithmically improved regularity criteria for the generalized Navier-Stokes and related equations," Kinetic and Related Models, vol. 6, no. 3, pp. 545-556, 2013.

[15] H. Kozono and Y. Taniuchi, "Bilinear estimates in BMO and the Navier-Stokes equations," Mathematische Zeitschrift, vol. 235, no. 1, pp. 173-194, 2000.

[16] H. Kozono, T. Ogawa, and Y. Taniuchi, "The critical Sobolev inequalities in Besov spaces and regularity criterion to some semi-linear evolution equations," Mathematische Zeitschrift, vol. 242, no. 2, pp. 251-278, 2002.

[17] T. Kato and G. Ponce, "Commutator estimates and the Euler and Navier-Stokes equations," Communications on Pure and Applied Mathematics, vol. 41, no. 7, pp. 891-907, 1988. 


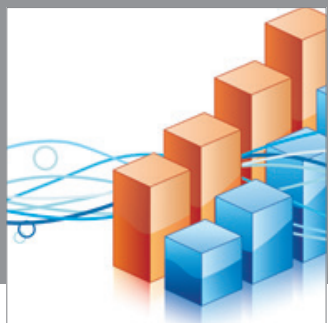

Advances in

Operations Research

mansans

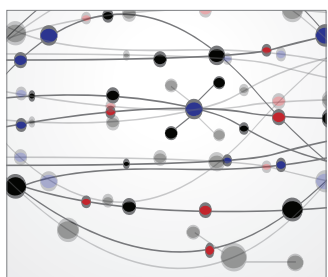

The Scientific World Journal
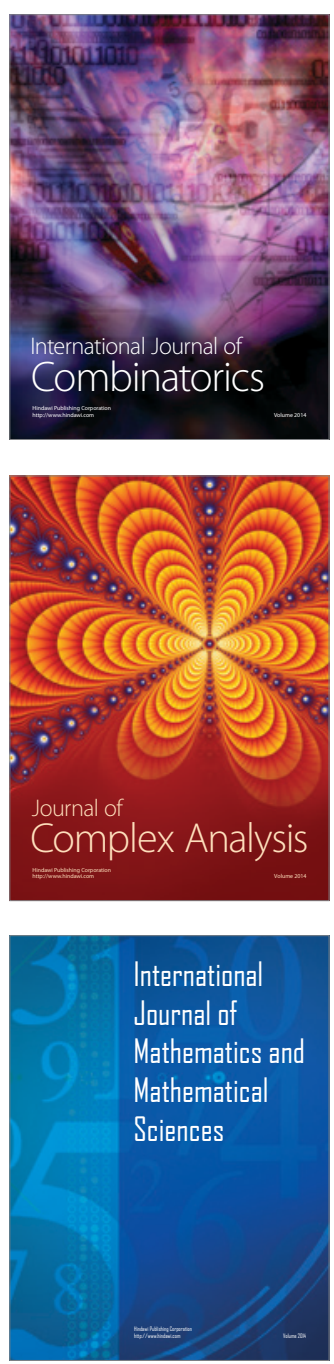
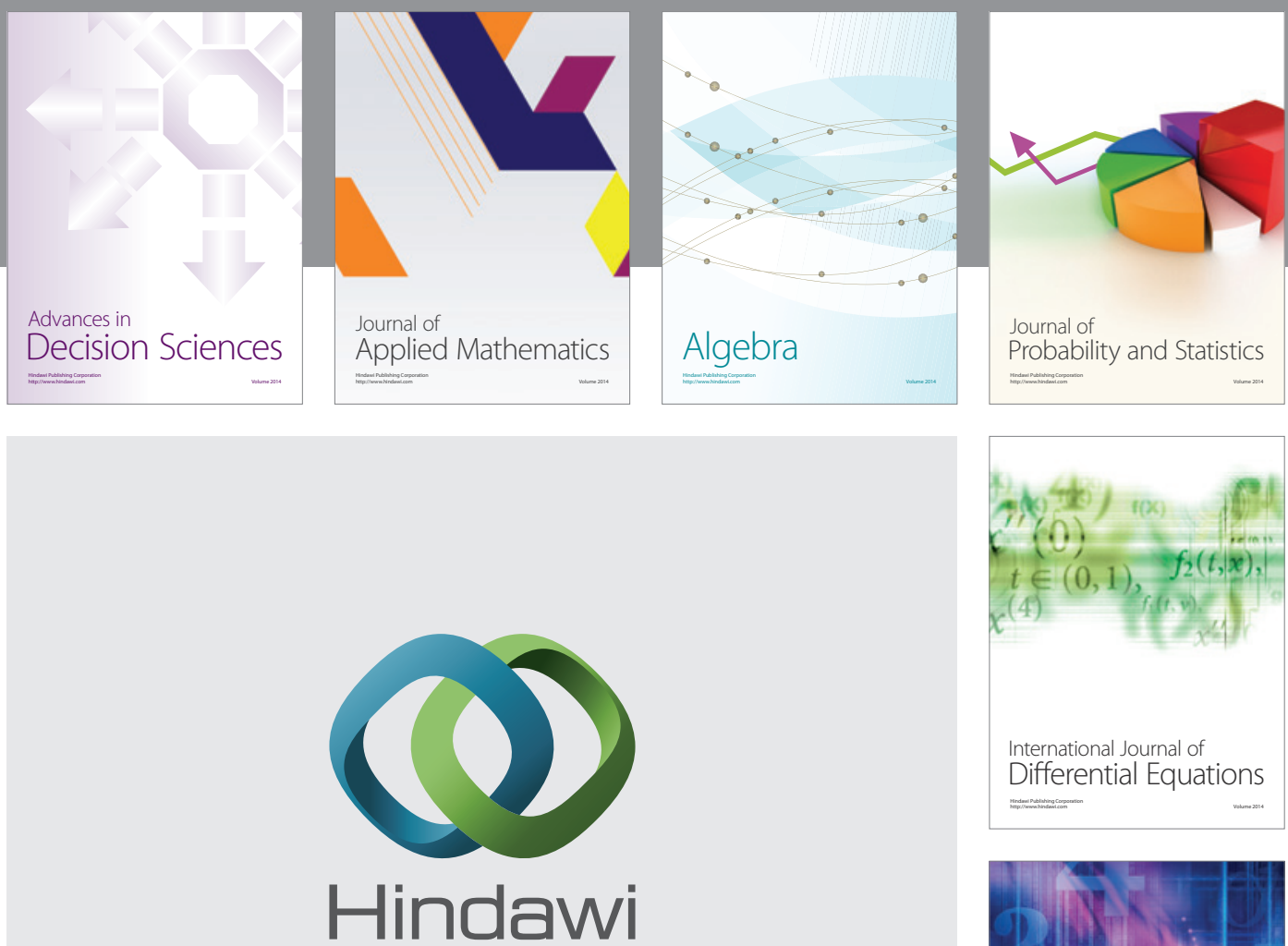

Submit your manuscripts at http://www.hindawi.com
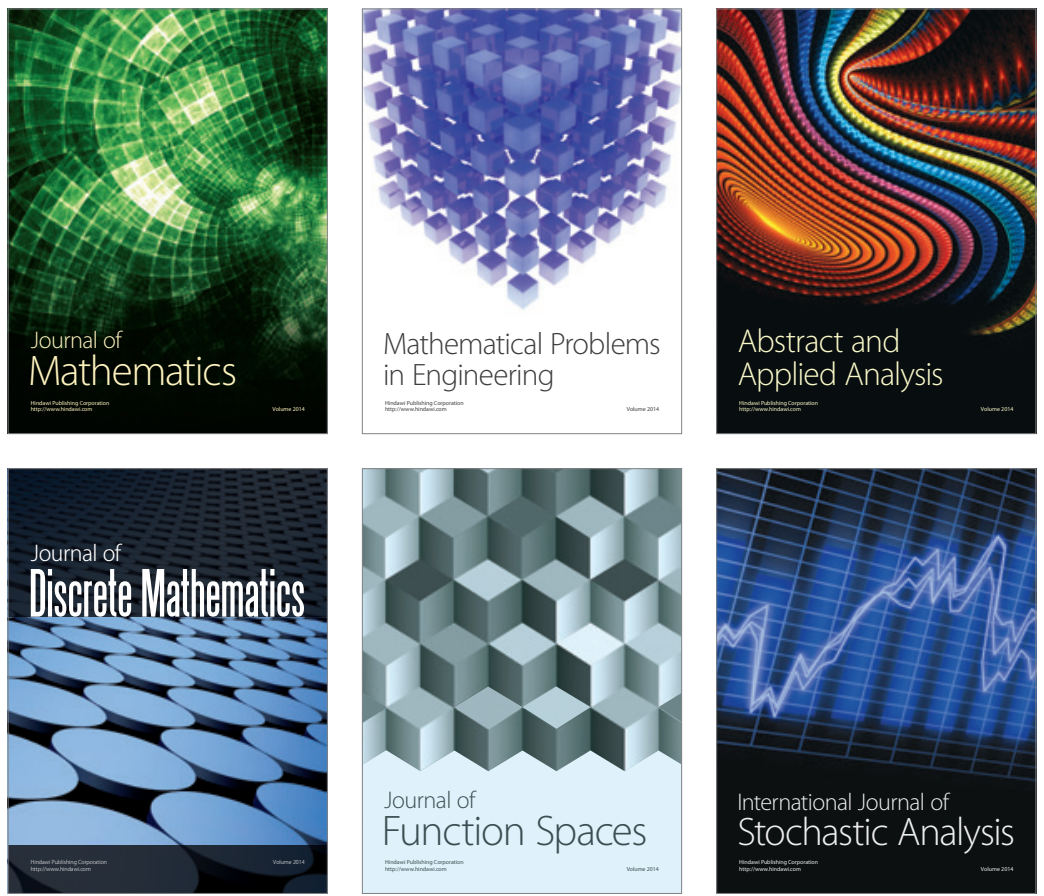

Journal of

Function Spaces

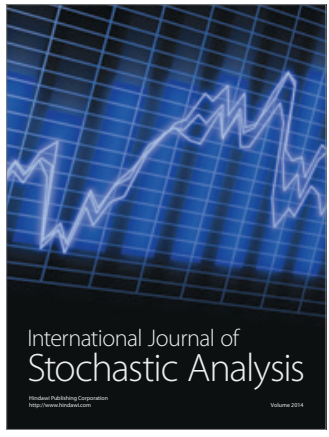

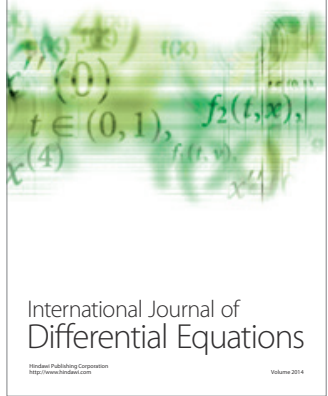
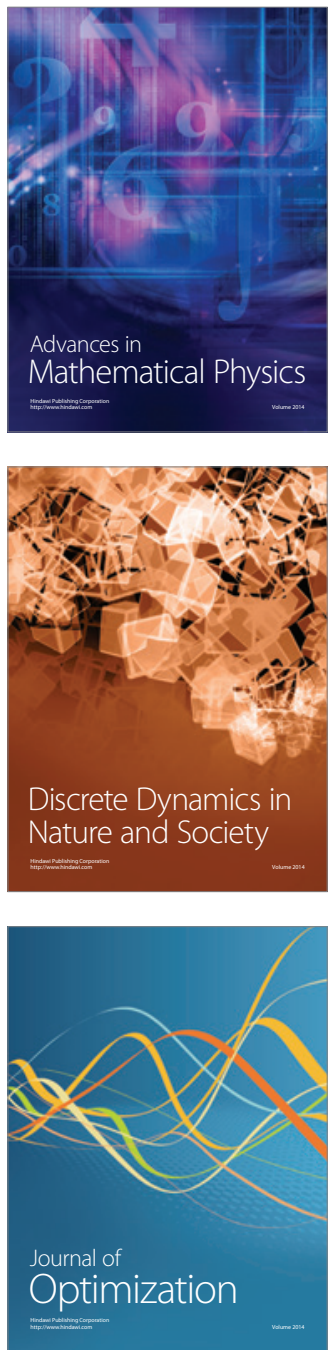